\title{
MODELOS HIPSOMÉTRICOS PARA UM POVOAMENTO JOVEM DE Khaya ivorensis A. Chev
}

\author{
HYPSOMETRIC MODELS FOR A Khaya ivorensis A. Chev YOUNG STAND \\ Lorena Stolle ${ }^{1}$, Diego Rodrigues Velozo ${ }^{2}$, Ana Paula Dalla Corte ${ }^{3}$, Roberto Carlos Sanquetta \\ Alexandre Beutling ${ }^{5}$ \\ 1,5 Universidade Federal de Mato Grosso do Sul, Chapadão do Sul, MS, Brasil - \\ lorenastolle@yahoo.com.br \& beutling.a@gmail.com \\ ${ }^{2}$ Analista de Qualidade Florestal, Veracel Celulose S.A., Eunápolis, BA, Brasil - \\ diego.velozo@veracel.com.br \\ 3,4 Universidade Federal do Paraná, Curitiba, PR, Brasil -anapaulacorte@gmail.com \& \\ carlos_sanquetta@hotmail.com
}

\section{RESUMO}

A medição de alturas das árvores em um povoamento florestal é considerada uma atividade onerosa, sendo usual medir a altura de apenas algumas árvores da parcela durante o inventário florestal. O objetivo deste trabalho consistiu em ajustar e testar modelos hipsométricos a fim de selecionar uma equação para estimar a altura das árvores de um povoamento de Khaya ivorensis (mogno africano) com 3 anos de idade. Os dados foram coletados em um povoamento de Mogno localizado no município de Chapadão do Sul, nordeste do estado do Mato Grosso do Sul, Brasil. Cinco modelos hipsométricos foram pré-selecionados e ajustados por regressão linear. A seleção da melhor equação foi realizada com base no coeficiente de determinação ajustado ( $R^{2} a j$.), erro padrão da estimativa (Syx\%), Critério de Informação de Akaike (AIC), valor de $\mathrm{F}$ e análise gráfica da distribuição dos resíduos. Os modelos selecionados apresentaram $\mathrm{R}^{2}$ aj. de 0,46, valores de Syx\% próximos de $11 \%$ e AIC variando de 36,7 a 38,6\%. A distribuição dos resíduos se mostrou homogênea e sem tendenciosidades. Baseado nas medidas de precisão, o modelo de Curtis foi considerado ligeiramente superior, porém os modelos de Henricksen e o Linear podem ser utilizados para estimativa da altura em função do diâmetro, pois apresentaram critérios estatísticos muito semelhantes.

PALAVRAS-CHAVE:Altura, Mensuração florestal, Mogno Africano.

\begin{abstract}
Tree heights measurement in a forest stand is onerous activity, and it is usual to measure the height of only a few trees in the forest inventory. The aim of this study was to fit hypsometric models to estimate the height of Khaya ivorensis (african mahogany) with 3 years old. The data were collected in a stand of African Mahogany in the city of Chapadão do Sul, northeastern Mato Grosso do Sul, Brazil. Five hypsometric models were pre-selected and fitted by linear regression. The selection of the best equation was based on the adjusted coefficient of determination $\left(R^{2} a j.\right)$, standard error of estimate (Syx\%), Akaike Information Criterion (AIC), F value and graphic residual analysis. The selected height-diameter models presented satisfactory results, with $\mathrm{R}^{2} \mathrm{aj}$. of 0.46 , Syx $\%$ around $11 \%$ and AIC between 36.7 and $38.6 \%$. The residual analysis was homogeneous and without bias. The Curtis model had the best adjustment, however Henricksen and Linear models can be used because they present very similar statistical criteria.
\end{abstract}

KEYWORDS: Height, Forest measurement, African Mahogany. 


\section{INTRODUÇÃO}

As plantações florestais no Brasil atingiram 7,84 milhões de hectares em 2016, porém apenas 7,5\% desta área são ocupadas por outras espécies que não sejam pinus e eucalipto (IBÁ, 2017). Segundo a Associação Brasileira de Produtores de Mogno Africano, os plantios de Khaya ivorensis A. Chev. ocupam cerca de dez mil hectares no Brasil (RIBEIRO et al., 2017).

Os primeiros plantios do mogno africano ocorreram na década de 1970 no Norte do país (RIBEIRO et al., 2017) visando a substituição da madeira do mogno brasileiro (Swietenia macrophylla King) devido principalmente ao controle exercido pelos órgãos ambientais ao comércio e exploração, assim como o uso predatório sem critérios de sustentabilidade (GROGAN et al., 2002). Poltronieri et al. (2000) citam que o mogno africano vem substituindo o mogno brasileiro em virtude da sua resistência contra o ataque da Hypsypila grandella Zeller.

Além disso, o aumento da demanda do mercado de madeira tropical tem conduzido a novos investimentos do plantio de mogno africano em todo o país (RIBEIRO et al., 2017).

Dada a importância que esta espécie vem se tornando no Brasil, verifica-se a necessidade de estudos de crescimento e produção por meio dos inventários florestais, os quais normalmente incluem a medição do diâmetro a $1,3 \mathrm{~m}$ de altura $(d)$, altura $(h)$ e volume das árvores $(v)$.

Segundo Machado e Figueiredo Filho (2003) a altura é a segunda variável independente utilizada nos métodos estimativos em tabelas de volume, funções de afilamento e em outras relações dendrométricas. Nos inventários florestais, as alturas das árvores não medidas dentro da parcela são estimadas por meio de uma relação hipsométrica expressa por uma equação ajustada (MACHADO \& FIGUEIREDO FILHO, 2003; SANQUETTA et al., 2014b).

A medição da altura de todas as árvores na parcela é uma atividade que requer um elevado tempo de dedicação, pois é muitas vezes uma tarefa de difícil execução devido a alguns fatores como: dificuldade de observar o ápice da árvore na presença de um sub-boque denso ou mesmo em plantios com espaçamentos pequenos; inclinação acentuada do terreno, e da operação inadequada dos equipamentos para medição de alturas (SANQUETTA et al., 2014b).

Schneider et al. (2009) cita que as relações biométricas, como a de diâmetro-altura, possibilitam obter valores estimados através de equações de regressão com o objetivo de reduzir tempo e custo na coleta de dados.

Diversos trabalhos utilizaram a relação hipsométrica para estimar a altura em povoamentos florestais principalmente de Pinus (SCHIMIDT, 1977; BLANCO JORGE, 1983; BARROS, et al., 2002; SENA et al., 2015; NICOLETTI et al., 2016), Eucalipto (COUTO \& BASTOS, 1987; ZANON et al., 1996; TOMÉ et al., 2007; RUFINO et al., 2010; MENDONÇA et al., 2015; SOUZA et al., 2017), Teca (ROSSI et al., 2011; PEREIRA et al.; 2014; SANQUETTA et al., 2015), Acácia negra (FINGER et al., 2000; SANQUETTA et al., 2014a), Imbuia (SANTOS et al., 2012), Bracatinga (BARTOSZECK, et al. 2004) e para povoamentos mistos de restauração florestal (SANQUETTA et al., 2017).

Silva et al. (2016) realizaram o ajuste de modelos hipsométricos para o Mogno Africano em Pirapora, no estado de Minas Gerais, e concluíram que há necessidade de maiores estudos para melhorar o ajuste dos modelos testados, porém os resultados encontrados são compatíveis com a origem seminal do povoamento, o qual caracterizou uma grande variabilidade nos dados.

Neste contexto, este trabalho teve como objetivo ajustar e comparar modelos hipsométricos para selecionar uma equação que sirva para estimar a altura das árvores em um povoamento de Khaya ivorensis (mogno africano), como forma de contribuir para os estudos recentes desta espécie no Brasil.

\section{MATERIAL E MÉTODOS}

\section{Área de estudo e coleta dos dados}

A área de estudo está localizada no município de Chapadão do Sul, no nordeste do estado de Mato Grosso do Sul (Microrregião de Cassilândia), Brasil.

Segundo a classificação de Köppen está sob influência do clima tropical de monção (Am), com precipitação média anual entre 1.600 e $1.900 \mathrm{~mm}$, temperatura média anual entre $22^{\circ} \mathrm{C}$ e $24^{\circ} \mathrm{C}$ e altitude média de $663 \mathrm{~m}$ (ALVARES et al., 2013).

Os dados foram coletados em um povoamento de 30 ha com mogno africano oriundo de mudas por sementes, com três anos de idade, e espaçamento de $5 \mathrm{~m}$ x $5 \mathrm{~m}$. Foram instaladas 16 parcelas circulares com área de $1.257 \mathrm{~m}^{2}$, distribuídas segundo o processo de amostragem inteiramente aleatório. Em cada parcela mensurou-se a circunferência à altura do peito (CAP) de todas as árvores e altura total das 10 primeiras árvores mais a altura das árvores dominantes, totalizando a 
medição de 412 indivíduos, cuja distribuição por classes de diâmetro e altura podem ser observadas na Tabela 1.

As medidas da CAP foram tomadas com o auxílio de uma fita métrica e para a medição de altura utilizou-se um clinômetro Haglöf.

Tabela 1. Distribuição dos indivíduos amostrados por classes de diâmetro e altura em um povoamento de mogno africano em Chapadão do Sul, MS.

\begin{tabular}{ccccc}
\hline \multirow{2}{*}{$\begin{array}{c}\text { Classes de } \\
\text { diâmetro }(\mathbf{c m})\end{array}$} & \multicolumn{3}{c}{ Classes de altura $(\mathbf{m})$} & Total \\
\cline { 2 - 4 } & $\mathbf{5}+\mathbf{8}$ & $\mathbf{8}+\mathbf{1 1}$ & $\mathbf{1 1}+\mathbf{1 3}$ & \\
\hline $5+10$ & 19 & 2 & & 21 \\
$10+15$ & 41 & 269 & 37 & 347 \\
$15+20$ & & 30 & 14 & 44 \\
\hline Total & 60 & 301 & 51 & 412 \\
\hline
\end{tabular}

\section{Ajuste e seleção dos modelos de relação hipsométrica}

Primeiramente foi efetuada uma análise descritiva dos dados de diâmetro a $1,3 \mathrm{~m}$ de altura do peito $(d)$ e altura para verificar o grau de homogeneidade dos dados e a presença de pontos discrepantes, e posteriormente foram avaliados os índices de correlação de Pearson $(r)$ para verificar a existência de correlação entre as variáveis a fim de prosseguir com o ajuste dos modelos. Para o modelo de Trorey (dupla entrada) foi verificada a existência de multicolinearidade entre as variáveis independentes por meio do Fator de Inflação de Variância (VIF).

Em seguida os dados sofreram ajuste por meio de regressão linear, onde foram testados cinco modelos de relação hipsométrica (aritméticos e logarítmicos) obtidos na literatura (Tabela 2).

Tabela 2. Modelos hipsométricos tradicionais testados para o ajuste dos dados de diâmetro e altura de um povoamento de mogno africano em Chapadão do Sul, MS.

\begin{tabular}{ccc}
\hline No & Denominação & Modelos \\
\hline 1 & Trorey & $\hat{h}=\beta_{0}+\beta_{1} d+\beta_{2} d^{2}$ \\
2 & Curtis & $\ln (\hat{h})=\beta_{0}+\beta_{1} 1 / d$ \\
3 & Stoffels \& van Soest & $\ln (\hat{h})=\beta_{0}+\beta_{1} \ln (d)$ \\
4 & Henricksen & $\hat{h}=\beta_{0}+\beta_{1} \ln (d)$ \\
5 & Modelo Linear & $\hat{h}=\beta_{0}+\beta_{1} d$ \\
\hline
\end{tabular}

Em que: $\ln =$ logaritmo neperiano; $\hat{h}=$ altura total estimada da árvore $(\mathrm{m}) ; d=$ diâmetro à $1,3 \mathrm{~m}$ de altura $(\mathrm{cm}) ;$ e $\beta_{0}, \beta_{1}$ e $\beta_{2}=$ coeficientes da regressão.
O critério de seleção para escolha da melhor equação (Tabela 3) obedeceu, inicialmente, ao maior coeficiente de determinação ajustado ( $R^{2}$ aj.), ao menor erro padrão da estimativa em porcentagem (Syx\%), o qual foi recalculado para as equações 2 e 3 (Tabela 2), devido à transformação logarítmica da variável dependente, menor valor do Critério de Informação de Akaike, maior valor de F e a análise gráfica dos resíduos (Tabela 3).

Tabela 3. Critérios de seleção para escolha da melhor equação para estimativa da altura em função do diâmetro para um povoamento de mogno africano em Chapadão do Sul, MS.

\begin{tabular}{|c|c|}
\hline Critério & Fórmula \\
\hline $\begin{array}{l}\text { Coeficiente de } \\
\text { determinação }\end{array}$ & $R^{2}=\frac{S Q \text { Regressão }}{S Q \text { Total }}$ \\
\hline $\begin{array}{c}\text { Coeficiente de } \\
\text { determinação ajustado }\end{array}$ & $R^{2} a j .=1-\left[\left(1-R^{2}\right)\left(\frac{n-1}{n-p}\right)\right]$ \\
\hline $\begin{array}{l}\text { Erro padrão da } \\
\text { estimativa }\end{array}$ & $S y x=\sqrt{\frac{\sum(h-\hat{h})^{2}}{n-p}}$ \\
\hline $\begin{array}{l}\text { Erro padrão da } \\
\text { estimativa \% }\end{array}$ & $S y x \%=\frac{S y x}{\bar{h}}$ \\
\hline $\begin{array}{c}\text { Critério de Informação } \\
\text { de Akaike }\end{array}$ & $A I C=n \ln \left(\frac{S Q \text { de Resíduos }}{n}\right)+2 p$ \\
\hline Resíduos & $r r \%=\frac{(h-\hat{h})}{h}$ \\
\hline
\end{tabular}

Em que: $S Q=$ soma de quadrados; $n=$ número de observações; $p$ = número de coeficientes da regressão; $h$ = altura observada; $\hat{h}=$ altura estimada; e $\bar{h}=$ altura média observada.

A discrepância logarítmica dos modelos 2 e 3 (Tabela 2) foi corrigida pelo Fator de Correção de Meyer. Por fim, avaliou-se o valor de $\mathrm{F}$ na análise de variância para verificar a existência da regressão e a significância dos seus coeficientes pelo teste $t$.

\section{Validação do modelo selecionado}

Do conjunto total de árvores mensuradas foram selecionadas 102 árvores (25\%) para compor uma amostra utilizada para a validação do modelo selecionado. Para a validação foi realizado o teste QuiQuadrado $(\chi 2)$ ao nível de $5 \%$ de probabilidade, onde se testou as seguintes hipóteses: Hipótese da nulidade: o modelo escolhido representa a relação hipsométrica dos dados; e Hipótese alternativa: o modelo escolhido não representa a relação hipsométrica dos dados. 


\section{RESULTADOS E DISCUSSÃO}

A análise descritiva dos dados utilizados no ajuste e na validação dos modelos de relação hipsométrica, mostra que as variáveis se apresentaram pouco heterogêneas (Tabela 4), representado pelos valores médios do coeficiente de variação (CV\%), segundo os critérios reportados por Pimentel-Gomes (1985). O diâmetro apresentou as menores dispersões e a altura dos dados de ajuste com maior variação (Tabela 4).

Tabela 4. Análise estatística descritiva das variáveis diâmetro $(d)$ e altura $(h)$ para um povoamento de mogno africano em Chapadão do Sul, MS.

\begin{tabular}{|c|c|c|c|c|c|c|}
\hline \multicolumn{2}{|c|}{ Variável } & \multirow{2}{*}{$\begin{array}{c}\text { Média } \\
13,3\end{array}$} & \multirow{2}{*}{$\begin{array}{c}\text { Desvio } \\
1,70\end{array}$} & \multirow{2}{*}{$\begin{array}{c}\text { Mínimo } \\
7,93\end{array}$} & \multirow{2}{*}{$\begin{array}{c}\text { Máximo } \\
16,55\end{array}$} & \multirow{2}{*}{$\begin{array}{r}\text { CV(\%) } \\
12,85\end{array}$} \\
\hline 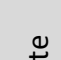 & $d$ & & & & & \\
\hline$<$ & $h$ & 9,4 & 1,44 & 5,50 & 13,10 & 15,29 \\
\hline \multirow{2}{*}{ 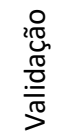 } & $d$ & 13,31 & 1,53 & 9,10 & 16,33 & 11,53 \\
\hline & $h$ & 9,59 & 1,15 & 7,20 & 12,30 & 11,94 \\
\hline
\end{tabular}

A matriz de correlação (Tabela 5) mostra que houve correlação significativa a $5 \% \quad(p$-valor $<0,05)$ entre as variáveis dependentes e independentes dos modelos testados, indicando que os modelos podem ser ajustados e testados para estabelecer uma relação hipsométrica com os dados coletados de Mogno Africano.

$O$ valor elevado de correlação $(r=0,99)$ entre as variáveis independentes $d$ e $d^{2}$ (Tabela 5) sugere a presença de multicolinearidade para o modelo 1 (Trorey), o qual foi evidenciado pelo VIF de 123,4 , onde um VIF > 10 indica a existência de efeitos graves de multicolinearidade.

Tabela 5. Matriz de correlação de Pearson entre as variáveis dependentes e independentes das equações ajustadas

\begin{tabular}{lccccc}
\hline \multirow{2}{*}{ Variáveis } & & \multicolumn{4}{c}{ Independentes } \\
\cline { 2 - 6 } & & $\boldsymbol{d}$ & $\boldsymbol{d}^{2}$ & $\mathbf{1 / \boldsymbol { d }}$ & $\boldsymbol{\operatorname { l n } ( \boldsymbol { d } )}$ \\
\hline \multirow{2}{*}{ Dependentes } & $h$ & $0,68^{*}$ & $0,67^{*}$ & & $0,68^{*}$ \\
& $\ln (h)$ & & & $-0,70^{*}$ & $0,70^{*}$ \\
Independentes & $d$ & & $0,99^{*}$ & & \\
\hline
\end{tabular}

Em que: * significativo ao nível de $5 \%$ de probabilidade.

As maiores correlações ocorreram com as variáveis transformadas, fato evidenciado também por Sanquetta et al. (2014a) e Souza et al. (2017), porém observam-se que todos os índices estão muito próximos entre si.

Os parâmetros estimados para os cinco modelos e as estatísticas de ajuste e precisão são apresentados na Tabela 6 onde se verifica que o valor de $F$ foi significativo a $5 \%$ para todos os modelos indicando a existência de regressão entre as variáveis dependentes e independentes. Porém os modelos 1 e 3 apresentaram coeficientes não significativos indicando que estes coeficientes poderiam ser retirados da equação ajustada. Neste trabalho, optou-se por eliminar estas equações, pois, além disso, a equação 1 (Trorey) apresentou problemas de multicolinearidade.

Os valores do coeficiente de determinação ajustado foram de 0,46 para todos os modelos restantes (2, 4 e 5), como mostra a Tabela 6. Segundo Campos e Leite (2006) normalmente a relação $h / d$ não é tão forte sendo muito comum que o valor do coeficiente de determinação seja menor que 0,8 .

Tabela 6. Coeficientes dos modelos ajustados e estatísticas de precisão.

\begin{tabular}{|c|c|c|c|c|c|c|c|}
\hline \multirow{2}{*}{ № } & \multicolumn{3}{|c|}{ Coeficientes dos Modelos } & \multirow{2}{*}{$\mathbf{F}$} & \multirow{2}{*}{$R^{2}$ aj. } & \multirow{2}{*}{ Syx\% } & \multirow{2}{*}{ AIC } \\
\hline & $\beta_{0}$ & $\beta_{1}$ & $\beta_{2}$ & & & & \\
\hline 1 & $-1,5179^{\text {ns }}$ & 1,1191 & $-0,0219^{\text {ns }}$ & $132,0^{*}$ & 0,46 & 11,2 & 38,7 \\
\hline 2 & 2,9679 & $-9,5974$ & & $307,3^{*}$ & 0,46 & 11,2 & 36,7 \\
\hline 3 & $0,1437^{\text {ns }}$ & 0,8100 & & $305,8^{*}$ & 0,48 & 11,3 & 38,0 \\
\hline 4 & $-8,6408$ & 7,0100 & & $264,6^{*}$ & 0,46 & 11,2 & 36,8 \\
\hline 5 & 1,8269 & 0,5722 & & $261,2^{*}$ & 0,46 & 11,3 & 38,6 \\
\hline
\end{tabular}

Em que: $n s$ = não significativo estatisticamente a $95 \%$ de probabilidade; $e^{*}=$ significativo ao nível de $5 \%$ de probabilidade

Sousa et al. (2013) encontraram valores semelhantes de $R^{2}$ aj. (entre 0,40 e 0,43 ) para os mesmos modelos em estudos de relação hipsométrica para Eucalyptus urophylla em regime alto fuste. Valores baixos de $\mathrm{R}^{2}$ também foram encontrados por Sanquetta et al. (2014a) ao definir uma relação hipsométrica para Acacia mearnsii e por Schikowski et al. (2014) para Cryptomeria japonica e Nicoletti et al. (2016) para Pinus taeda.

Silva et al. (2016) encontraram valores de $R^{2} a j$. próximos de 0,7 para um estudo com Khaya ivorensis com idade entre 30 e 47 meses plantadas em espaçamento $4 \mathrm{~m}$ × $3 \mathrm{~m}$ no município de Pirapora, Minas Gerais. Os autores sugerem que o valor baixo encontrado para o coeficiente de determinação se deve ao fato de que o plantio de Mogno é proveniente de sementes, com maior 
variabilidade nos dados.

O erro padrão para os modelos deste estudo ficou próximo de $11 \%$, o que pode ser considerado como uma boa precisão dada à baixa correlação entre as variáveis e o baixo coeficiente de determinação das equações. Nos estudos de relação hipsométrica encontrados na literatura são encontrados valores de Syx\% acima de 10\% (SANQUETTA et al., 2014a) para Acácia, para Teca (SANQUETTA et al., 2015), para Eucalipto (RETSLAFF et al., 2015; SANTOS et al., 2014) e em alguns casos menor que $10 \%$ para Pinus (NICOLETTI et al., 2016).Silva et al. (2016) encontraram erros em torno de $2 \mathrm{~m}$ na estimativa da altura por relações hipsométricas para Khaya ivorensis em Pirapora.

Os valores de AIC ficaram próximos entre os modelos restantes (2, 4 e 5), sendo o do modelo 2 (Curtis), levemente superior, este modelo também apresentou o maior valor de F.A distribuição gráfica dos resíduos mostrou-se semelhante para todas as equações, onde foi verificada a ausência de tendência nas estimativas das alturas e uma dispersão homogênea dos resíduos (Figura 1).

Analisando-se todos os resultados apresentados podese afirmar que o modelo de Curtis é levemente superior aos demais por apresentar menor valor de AIC e maior valor de $\mathrm{F}$, sendo este modelo adequado para estimar a altura total do plantio de mogno africano. No entanto, observa-se que os modelos de Henricksen e Linear apresentaram estatísticas de seleção muito semelhantes no ajuste da relação hipsométrica.

O teste Qui-Quadrado aplicado nos dados de validação indicou que as alturas observadas em campo não diferem estatisticamente das alturas estimadas pela equação ajustada do modelo de Curtis, onde para este conjunto de dados o coeficiente de determinação ajustado ficou um pouco maior $(0,54)$, o erro padrão da estimativa diminuiu (10\%).

\section{CONCLUSÕES}

A relação hipsométrica para este povoamento de Mogno Africano com 3 anos de idade não é forte, pois todas as equações testadas apresentaram coeficientes de determinação próximos de 0,4.

O erro padrão da estimativa e a análise de resíduos mostram que os modelos de Curtis, Henricksen e Linear podem ser utilizadas para estimativa da altura total com erros em torno de $11 \%$ e sem tendenciosidades.

O modelo de Curtis apresenta leve superioridade para as estimativas das alturas, onde a validação demonstrou que as estimativas são confiáveis com erro de $10 \%$.

Curtis
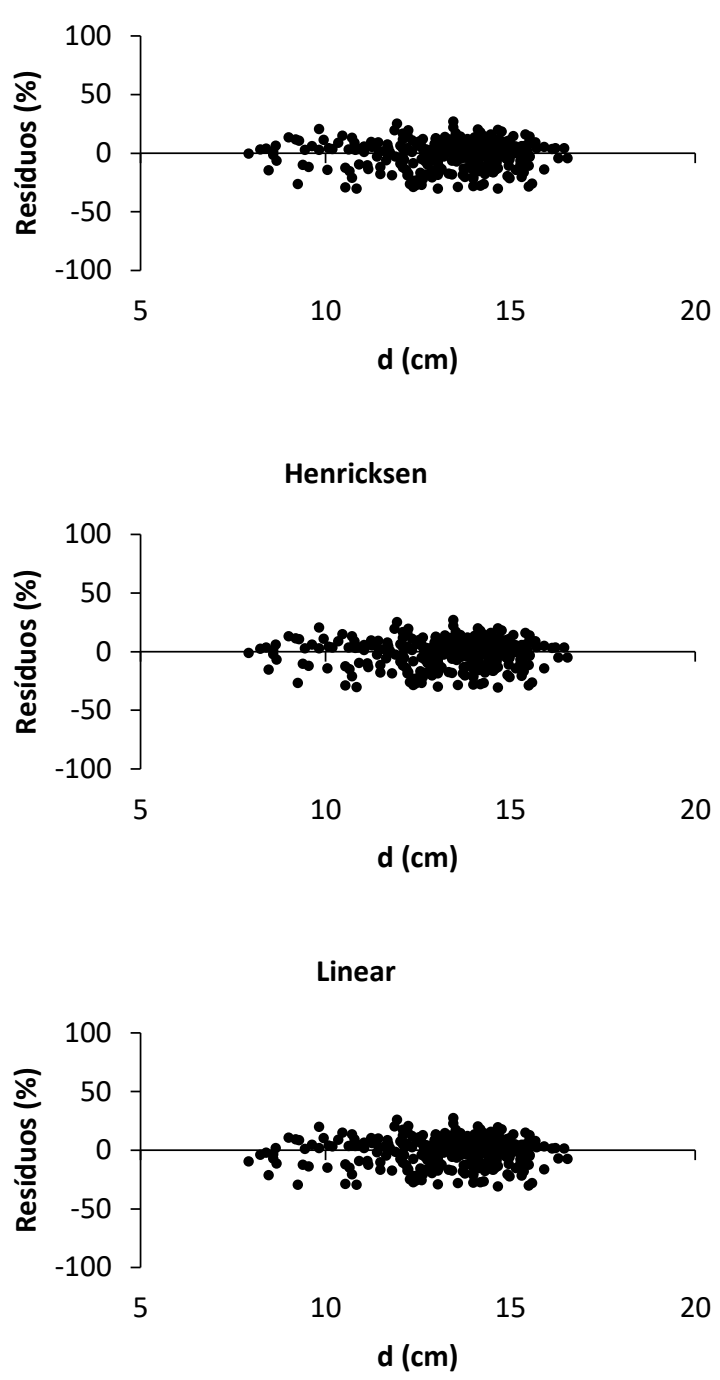

Figura 1. Distribuição de resíduos em função do diâmetro (d) das equações hipsométricas ajustadas para um povoamento de Mogno Africano em Chapadão do Sul, MS.

\section{REFERÊNCIAS}

ALVARES, C. A. et al. Köppen's climate classification map for Brazil. Meteorologische Zeitschrift, v. 22, n. 6, p. 711-728, 2013.

BARROS, D. A. et al. Comportamento de modelos hipsométricos tradicionais e genéricos para plantações de Pinus oocarpa em diferentes tratamentos. Boletim de Pesquisa Florestal, n. 45, p. 3-28, 2002.

BARTOSZECK, A. C. P. S. et al.Dinâmica da relação hipsométrica em função da idade, do sítio e da densidade inicial de povoamentos de bracatinga da Região Metropolitana de Curitiba, PR. Revista Árvore, v. 28, n. 4, p. 517-533, 2004.

BLANCO JORGE, L. A. Equações de relação hipsométrica para povoamentos de Pinus elliottii E. na Floresta Nacional de Três 
Barras, SC. Brasil Florestal, n. 56, p. 41-47, 1983.

CAMPOS, J. C. C.; LEITE, H. G. Mensuração Florestal: Perguntas e Respostas. 2 ed. Viçosa: UFV, 2006. 470 p.

COUTO, H. T. Z.; BASTOS, N. L. M.; Modelos de equações de volume e relações hipsométricas para plantações de Eucalyptus no Estado de São Paulo. IPEF, n. 37, p. 33-44, 1987.

FINGER, C. A. G. et al. Curvas de altura-diâmetro de acácia negra (Acacia Mearnsii de Wild). Ciência Rural, v. 30, n. 3, p. 387-391, 2000.

GROGAN, J. et al. Mogno na Amazônia Brasileira: Ecologia e perspectivas de manejo. Belém: Imazon, 2002. 64 p.

IBÁ - Indústria Brasileira de Árvores. Relatório IBA 2017. São Paulo: IBÁ, 2017. 80 p.

MACHADO, S. A.; FIGUEIREDO FILHO, A. Dendrometria. Curitiba: Editado pelos autores, 2003. 309 p.

MENDONÇA, A. R. et al. Modelos hipsométricos generalizados mistos na predição da altura de Eucalyptus sp. Cerne, v. 21, n. 1, p. 107-115, 2015.

NICOLETTI, M.F. et al. Relação hipsométrica para Pinus taeda L. em diferentes fases do ciclo de corte. Floresta e Ambiente, v. 23, n. 1, p. 80-89, 2016.

PEREIRA, K. D. et al. Ajuste de modelos hipsométricos para árvores de Tectona grandis L. f. no município de Mojú, Pará. Enciclopédia Biosfera, v. 10, n. 18, p. 181-189, 2014.

PIMENTEL-GOMES, F. Curso de estatística experimental. 12. ed. Piracicaba: Livraria Nobel, 1985. 467p.

POLTRONIERI L. S. et al. Identificação de doenças em mognoafricano no Estado do Pará. Belém: Embrapa Amazônia Oriental, 2000. 13 p. Circular Técnica, n. 18.

RETSLAFF, F.A.S. et al. Curvas de sítio e relações hipsométricas para Eucalyptus grandis na região dos Campos Gerais, Paraná. Cerne, v. 21, n. 2, p. 219-225, 2015.

RIBEIRO, A. et al. O cultivo do mogno africano (Khaya spp.) e o crescimento da atividade no Brasil. Floresta e Ambiente, v. 24, 2017.

ROSSI, A. S. et al.Relação hipsométrica e crescimento de Tectona grandis L. f. no município de Monte Dourado, Pará. Scientia Forestalis, v. 39, n. 91, p. 301-307, 2011.

RUFINO, R. F. et al. Ajuste de modelos hipsométricos para um povoamento de eucaliptos conduzido sobre o sistema de rebrota. Enciclopédia Biosfera, v. 6, n. 10, p. 1-10, 2010.

SANQUETTA, C. R. et al.Relação hipsométrica em inventários pré-corte em povoamentos de Acacia mearnsii De Wild. Científica, v. 42, n. 1, p. 80-90, 2014a.

SANQUETTA, C. R. et al. Inventários Florestais: planejamento e execução. 3. ed. Curitiba: Multi-Graphic Gráfica e Editora, 2014b. 406 p.
SANQUETTA, C. R. et al. Estimativa da altura e do volume em povoamentos jovens de restauração florestal em Rondônia. Biofix Scientific Journal, v. 2, n. 2, p. 23-31, 2017.

SANQUETTA, M. N. I. et al. Ajuste de equações hipsométricas para a estimação da altura total de indivíduos jovens de teca. Científica, v. 43, n. 4, p. 400-406, 2015.

SANTOS, A. T. et al. Equação de volume e relação hipsométrica para plantio de Ocotea porosa. Pesquisa Florestal Brasileira, v. 32, n. 69, p. 13-21, 2012.

SANTOS, M. L. et al. Estimativa da relação hipsométrica para um povoamento de Eucalyptus "urograndis" no município de Mojú, nordeste paraense. Enciclopédia Biosfera, v. 10, n. 19, p. 10391048, 2014.

SCHNEIDER, P. R. et al. Análise de regressão aplicada a Engenharia Florestal. 2. ed. Santa Maria: FACOS-UFSM, 2009. $294 \mathrm{p}$.

SCHIKOWSKI, A. B. et al. Ajuste de modelos hipsométricos para povoamento de Cryptomeriajaponica (Thunb. ex L. f.) D. Don no Paraná.Enciclopédia Biosfera, v. 10, n. 19. p. 70-77, 2014.

SCHIMIDT, P. B. Determinação indireta da relação hipsométrica para povoamentos de Pinus taeda, L. Floresta, v. 8, n. 1, p. 2427, 1977.

SENA, A. L. M. et al. Modelos lineares com uso de covariantes para relação hipsométrica de duas espécies de pinus tropicais. Ciência Florestal, v. 25, n. 4, p. 969-980, 2015.

SILVA, L. F. et al. Equações hipsométricas, volumétricas e de crescimento para Khaya ivorensis plantada em Pirapora. Floresta e Ambiente, v. 23, n. 3, p. 362-368, 2016.

SOUSA, G. T. O. et al. Relações hipsométricas para Eucalyptus urophylla conduzidos sob regime de alto fuste e talhadia no Sudoeste da Bahia. Scientia Plena, v. 9, n. 4., p 1-7, 2013.

SOUZA, A. S. et al. Modelagem da relação hipsométrica para um povoamento híbrido de eucalipto na Amazônia Brasileira. Biofix Scientific Journal, v. 2, n. 1, p. 44-53, 2017.

TOMÉ, M. et al. Relação hipsométrica geral para Eucalyptus globulus Labill. em Portugal. Silva Lusitana, v. 15, n. 1, p. 41-55, 2007.

ZANON, M. L. B. et al. Funções para descrever a relação altura diâmetro de Eucalyptus dunnii Maiden. Ciência Rural, v. 26, n. 1, p. 87-90, 1996. 